\title{
Daytime variations of foE connected to earthquakes
}

\author{
E. V. Liperovskaya ${ }^{1}$, V. V. Bogdanov ${ }^{2}$, P.-F. Biagi ${ }^{3}$, C.-V. Meister ${ }^{4}$, and V. A. Liperovsky ${ }^{1}$ \\ ${ }^{1}$ Institute of Physics of the Earth of the Russian Academy of Sciences, Bolshaya Gruzinskaya 10, 123995 Moscow, Russia \\ ${ }^{2}$ Institute of Cosmophysical Research and Radio Wave Propagation, Far Eastern Branch of the Russian Academy of Sciences, \\ 684034 Petropavlovsk-Kamchatsky, Russia \\ ${ }^{3}$ Physics Department, University of Bari, 70126 Bari, Italy \\ ${ }^{4}$ Institut für Kernphysik, Technische Universität Darmstadt, Schlossgartenstr. 9, 64289 Darmstadt, Germany
}

Received: 15 October 2010 - Revised: 15 March 2011 - Accepted: 23 May 2011 - Published: 28 June 2011

\begin{abstract}
In the present work it is shown that, in accordance with the observations of the vertical sounding station "Tashkent", the critical foE-frequency of the daytime E-layer increases about one day before winter-earthquakes with magnitudes $M>5$ and depths of the epicentre of $h<60 \mathrm{~km}$, which appeared at distances of $R<2000 \mathrm{~km}$ from the station. The reliability of the result is larger than $99 \%$. The phenomenon is not observed for summer-earthquakes. It seems to be determined by the atmospheric wind system. Further, the variations of the foE-frequency are compared with possible simultaneous variations of the critical frequency foF 2 of the F2-layer. First results show that only very large changes of the ionisation density in the E-layer influence the ionisation density in the F-region. Therefore, no synchronous growth of the foE- and foF2-frequencies 1-2 days before seismic shocks could be observed.
\end{abstract}

\section{Introduction}

The E-region of the ionosphere is situated at $h \approx 90-140 \mathrm{~km}$ above the Earth's surface. Its ionisation density amounts to $N \lesssim 10^{5} \mathrm{~cm}^{-3}$ at daytime, and decreases by about $3-4$ orders of magnitude at night (Antonova et al., 1996). The midlatitudinal daytime E-layer is mainly formed by the ionisation of neutral $\mathrm{O}_{2}$-molecules by two close EUV lines with wavelengths of $977 \AA$ (CIII) and $1025.7 \AA$ (HLy $\beta)$, providing $80-90 \%$ of the total ionisation rate (Mikhailov et al., 2007). The remainder of the ions is provided by the $\mathrm{x}$-ray radiation with wavelengths below $100 \AA$ (Ivanov-Kholodny et al., 1976). The critical frequency foE of the daytime E-layer is generally described by a classical theory already introduced by Chapman in the 1930s (Chapman, 1931). The fre-

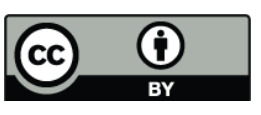

Correspondence to: C.-V. Meister (c.v.meister@ skmail.ikp.phyik.tudarmstadt.de) quency, above all, depends on the position of the Sun above the horizon, and of course on the solar activity. Additionally, it is modified by the concentration of the $\mathrm{O}_{2}$-molecules and its altitudinal scale, as well as by the ionisation and absorption cross sections of the material (Mikhailov et al., 2007). Thus, the behavior of foE is, above all, determined by the daily and seasonal changes of the solar activity, and also by the 11-yr and 27-days solar cycles. Additionally, the frequency is weakly influenced by disturbances of the geomagnetic field, by electrical disturbances propagating from the F-layer downwards, as well as by variations of the electric field and perturbations of acoustic nature propagating upwards from the neutral atmosphere. The influence of meteorological effects on the E-layer, and particularly on the foEfrequency, was studied by Danilov (1989). One may expect that electrical, acoustic, and acoustico-gravity disturbances occurring in the lower atmosphere during earthquake preparation times influence the variations of the foE-frequency. But as the foE-frequency, compared with other characteristic frequencies of the ionosphere, is rather stable with respect to external influences, and as its changes caused by disturbances of non-solar nature amount only to about one percent, it was considered to be only weakly sensitive to earthquake preparation processes. Therefore, the foE-frequency was only seldom analysed searching for seismo-ionospheric effects. Only in the work by Ivan-Kholodnyi and Tchertoprud (1998), an increase of foE about two days before some $(\approx 20)$ strong earthquakes was mentioned. On the other side, in some works (Liperovskaya et al., 2006, 2008, 2009) it was already concluded that 3-5 days before strong earthquakes the critical frequency foF2 of the F2-layer is somewhat modified.

The aim of the present work is the statistical proof of seismo-ionospheric effects in the foE-variations, attempting to define under which conditions the characteristic foEfrequency increases 1-2 days before earthquakes. Further, a first comparison of the variations of the foE-frequency with

Published by Copernicus Publications on behalf of the European Geosciences Union. 
simultaneous variations of the critical frequency foF 2 of the F2-layer before earthquakes is presented.

\section{Method of analysis}

In the present work, effects of earthquakes on the Elayer and F-layer of the ionosphere before seismic shocks are investigated. Therein, earthquakes with magnitudes $M>5$, epicentres situated at a distance from the vertical sounding station smaller than $R=2000 \mathrm{~km}$, and a depth of the source $h<60 \mathrm{~km}$ are taken into account. Data from the "Tashkent" station in Middle Asia (latitude $-\varphi=$ $41.3^{\circ} \mathrm{N}$, longitude $-\lambda=69.6^{\circ} \mathrm{E}$ ), which are published online (http://spidr.ngdc.noaa.gov/spidr/), are used.

For the study of seismo-ionospheric phenomena in the Elayer of the ionosphere, variations of the critical daytime foE-frequency are considered. The hours from 11:00 LT to 17:00 LT are taken as daytime, as the time when the degree of ionisation of the ionosphere is at maximum and the dependence of the degree of ionisation on the time is not so strong as in the other hours. For the analysis, the data averaged over the introduced interval of 11:00-17:00 LT, foE $\mathrm{day}_{\text {are used. }}$ foE $E_{\text {day }}$ is found when at least four values of the averages over the six daytime hours exist.

To exclude the seasonal dependence of the foE $\mathrm{day}_{\text {-values, }}$ the values of foE $_{11 \text { days, }}$, calculated using linear interpolation over 11 days (from day $(-5)$ until day $(+5)$ ), were subtracted from the values of $f_{\mathrm{day}}, \Delta E(i)=f o E_{\mathrm{day}}(i)-$ $f o E_{11 \text { days }}(i)$. The short time interval of 11 days is selected to also decrease the influence of the 27-days solar cycle. It is not possible to exclude the seasonal dependence completely. But, as will be shown below, the seasonal dependence remaining after the described averaging procedure is much smaller than the change of $\Delta E(i)$ by the ionospheric earthquake preparation processes.

From the obtained $\Delta E(i)$-values, all variations which have an absolute value larger than $2 \mathrm{MHz}$ are then excluded. The number of such values is about $1 \%$ of the total data (84 days of a total of 6425 days). The main part of the excluded data is related to strong geomagnetic and solar disturbances. Spikes in the $\Delta E(i)$-values also occur, the reason for which is unknown. It is reasonable to also omit these data before one calculates the mean square deviation $\operatorname{std}(\Delta E(i))$ for 11 days. Further, the $\Delta E(i)$-values are normalized by the mean square deviation $\operatorname{std}(\Delta E(i))$. As a result, every analysed day is characterized by a normalized function foEnorm $(i)=\Delta E(i) / \operatorname{std}(\Delta E(i))$.

The ionospheric effects before earthquakes are searched for in front of the background of solar and geomagnetic disturbances. Thus, only such days are considered in which the solar and geomagnetic disturbances are not too large. An accepted opinion about what is "too large" does not exist. In the present work, days with a Wolf number $W>150$ are neglected. As ionospheric changes may continue some days

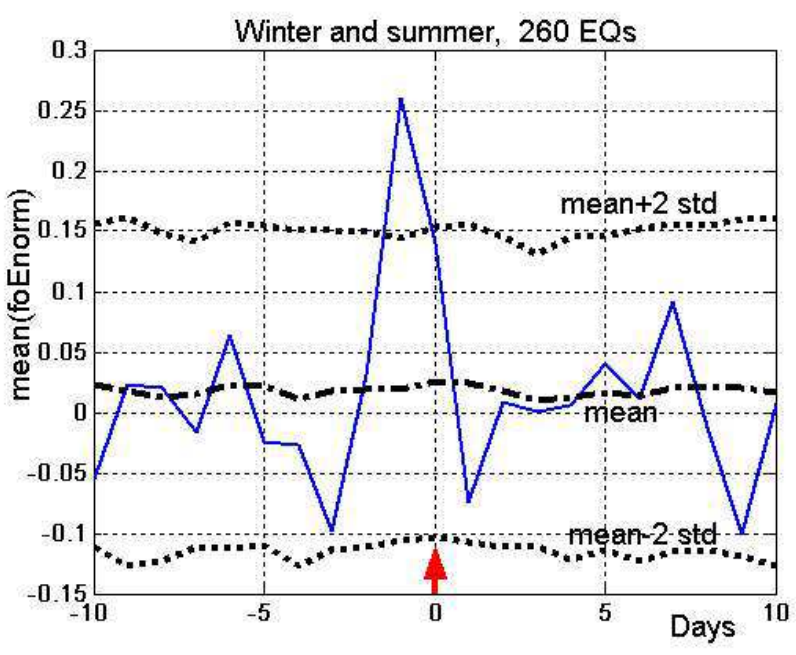

Fig. 1. Result of the superposition of epoches for the foEnormvalues for earthquakes with $M>5, R<2000 \mathrm{~km}$, and $h<60 \mathrm{~km}$. The superposition time equals 20 days. The red arrow shows the day zero of the earthquakes. The mean value of foEnorm $(i)$ for the day $i$ during seismo-active times is presented by the solid blue line. The dash-dotted line designates the mean value of foEnorm for all data considered. The dotted lines show the $95 \%$-interval of reliability obtained using the Monte-Carlo method.

after strong disturbances, days with a $\Sigma K_{p}$-index larger than 30 are also not taken into account.

Finally, having performed the data evaluation as described above, excluding days with strong solar and geomagnetic disturbances as previously described was inserted for clarity, the superposition of epoches method (Ambroz, 1979) is applied to earthquakes with magnitudes $M>5$, distances from the station $R<2000 \mathrm{~km}$, and focal depths $h<60 \mathrm{~km}$.

\section{Seismo-ionospheric effects in the E-layer}

Results of the analysis of the foE-data are shown in Fig. 1. The decrease of the mean foEnorm-value three days before the (260 studied) earthquakes (day (-3)) and the increase of the value one day before the seismic shocks (day $(-1)$ ) are demonstrated in Fig. 1. The decrease on day $(-3)$ seems to be random. First, it is unnatural to suggest that a seismoionospheric effect is so strongly localized in time a few days before an earthquake. Second, the effect is unstable: studying the dependence of the decrease of foEnorm on the different years, the casual character of the decrease can be seen.

Next, the seasonal dependence of foEnorm on day $(-1)$ before the seismic shock is considered. Figure 2 shows that the increase of foEnorm one day before a seismic shock is observed only in winter. In contrast, no effect is found during the summer months.

Thus, only earthquakes occurring in winter (from October till April, in the months 1, 2, 3, 4, 10,11, 12) are studied here. 
$260 \mathrm{EQs}, \mathrm{M}>5, \mathrm{R}<2000 \mathrm{~km}, \mathrm{~h}<60 \mathrm{~km}$

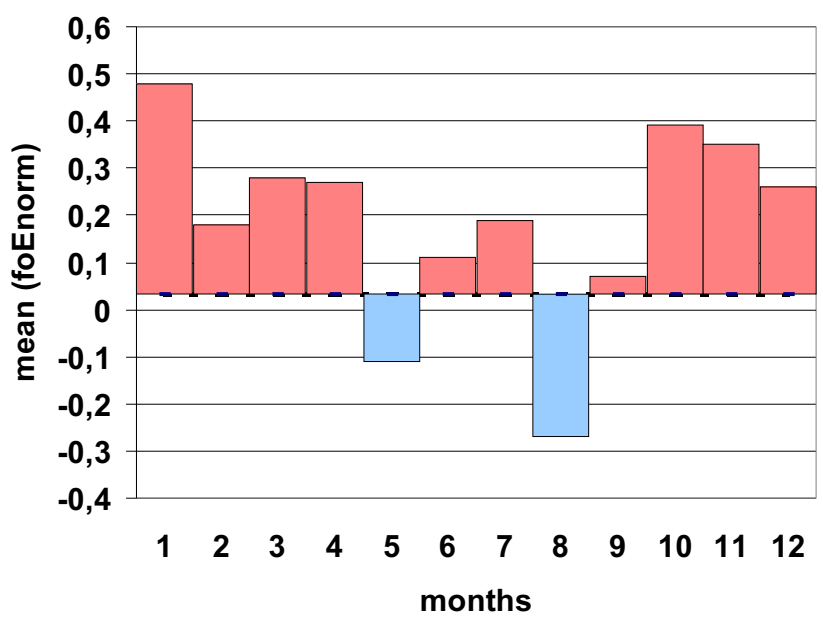

Fig. 2. Seasonal dependence of foEnorm one day before an earthquake shown by its monthly average values. The dash-dotted line presents the mean value for all days $(-1)$ of all data considered.

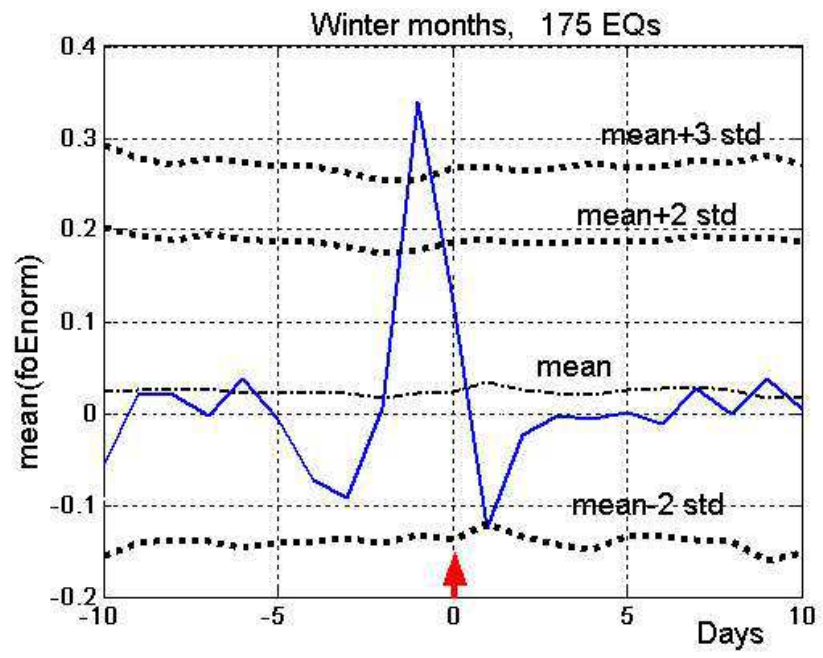

Fig. 3. Result of the superposition of epoches for foEnorm-values for winter-earthquakes with $M>5, R<2000 \mathrm{~km}$, and $h<60 \mathrm{~km}$. The superposition time equals 20 days. The red arrow shows the day zero of the earthquakes. The mean value of $f_{\text {foEnorm }}(i)$ for the day $i$ during seismo-active times is presented by the solid blue line. The dash-dotted line designates the mean value of foEnorm for all data considered. The dotted lines show the $95 \%$-levels ( 2 std.) and $99 \%$-levels ( 3 std.) of reliability obtained using the method of modeling random processes.

The increase of foEnorm on the day $(-1)$ before winterearthquakes with magnitudes $M>5$, distances $R<2000 \mathrm{~km}$, and depths $h<60 \mathrm{~km}$ is considerable. It is larger than $3 \mathrm{std}$ for a superposition interval of 20 days (see Fig. 3). At a superposition time of 100 days (from day -50 till day +50 ),

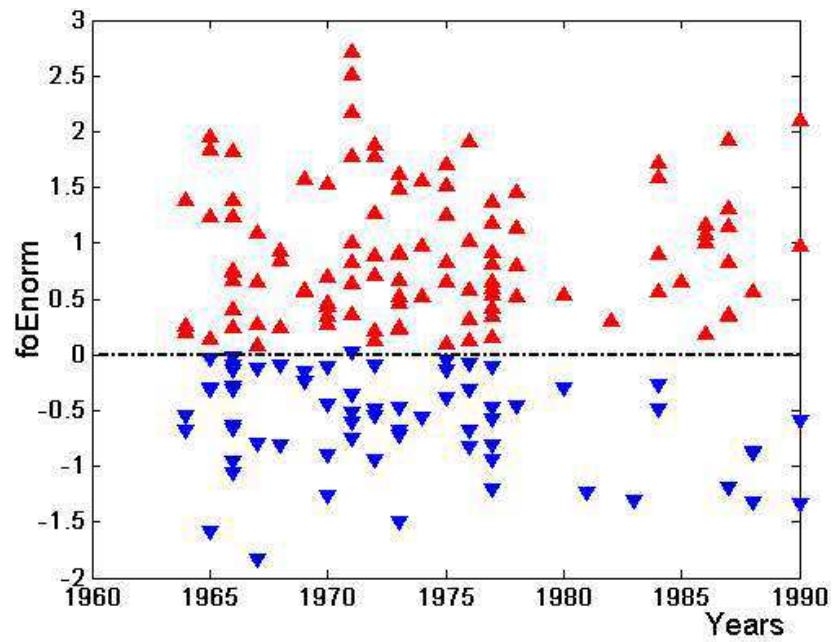

Fig. 4. Variations of foEnorm on the day $(-1)$ of all winterearthquakes in dependence on the year. Every earthquake is presented by a triangle. It is to be seen, that in all the years the number of earthquakes with foEnorm larger than the mean value is larger than the number of seismic shocks with foEnorm smaller than the average.

one even finds an increase larger than 4 std. This effect is rather stable, meaning it does not depend on the different years. Figure 4 shows the behaviour of foEnorm in the years from 1964 till 1988. From these results one may conclude that the increase of foEnorm on the day $(-1)$ is observed for the whole considered time interval. In total, there are analyzed data of 119 earthquakes for the day $(-1)$. Dividing the 119 earthquakes into three groups according to growing time, one finds for the first 40 events a mean value of 0.27 , for the group 2 of 40 earthquakes a mean value of 0.51 , and for the third group of 39 earthquakes a mean value 0.32 .

Further, the dependence of foEnorm of the day $(-1)$ on the distance between the earthquake epicentre and the sounding station is studied (Fig. 5). From Fig. 5 one can see a tendency that the maximum increase of foEnorm occurs at distances of $800-1600 \mathrm{~km}$ from the epicentre.

Considering the dependence of the seismo-ionospheric effect on the location of the epicentre for winter-earthquakes, it is found that foEnorm on the day (-1) is anisotropic (Fig. 6). In the case of earthquakes to the west of Tashkent, on day $(-1)$, one finds an increase of foEnorm for 44 seismic shocks and a decrease for 26 shocks. But for seismic events east of Tashkent, an increase on day $(-1)$ is observed in 28 cases, and a decrease occurs in 22 events. Earthquakes with an epicentre east of the sounding station mainly contribute to the increase of foEnorm. 


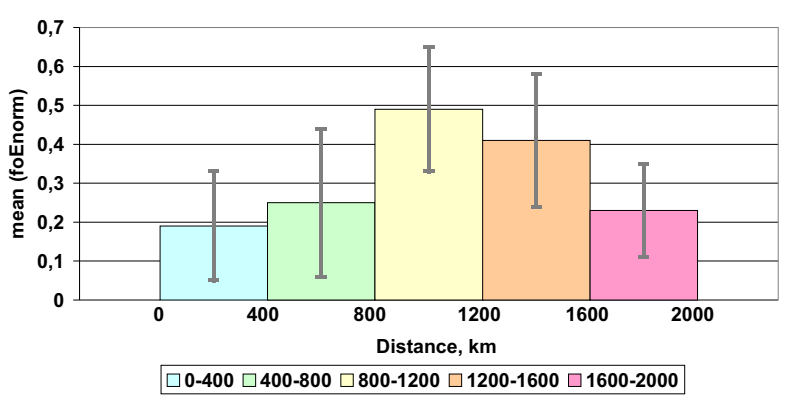

Fig. 5. Histogram showing the dependence of foEnorm on the distance from the epicentre. Distances are given in kilometers. The error bars describing \pm std are also inserted. The values of foEnorm are found by averaging all earthquakes with a distance which is within the interval shown in the inset.

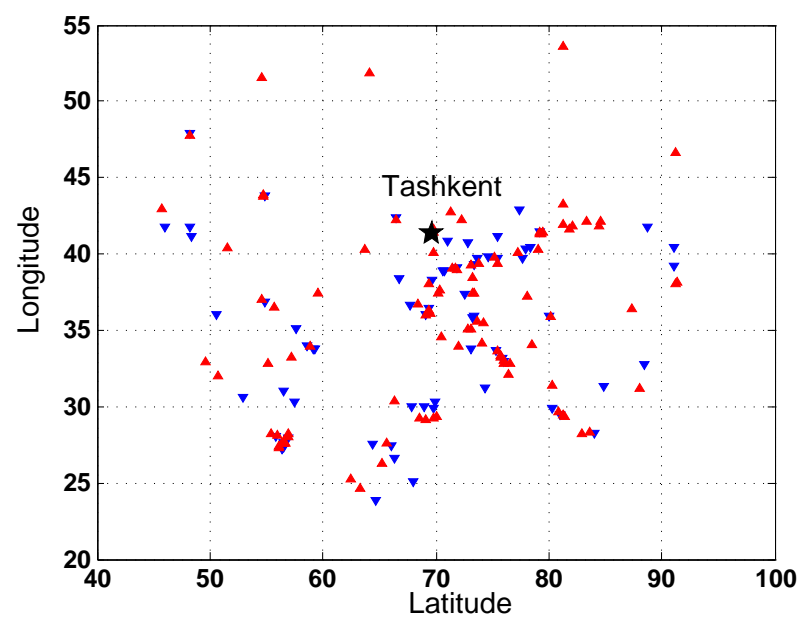

Fig. 6. Position of the earthquake epicentres relative to the vertical sounding station "Tashkent". Red points show earthquakes for which foEnorm on the day $(-1)$ is larger than the mean value, blue points designate earthquakes with a foEnorm-value on day $(-1)$ smaller than the mean value

\section{Seismo-ionospheric effects in the F-layer}

In the following, the results obtained in the present paper for the foE-frequency will be compared with the behaviour of the foF2-frequency obtained at the same time with the same vertical sounding station "Tashkent".

The formation mechanism of the mid-latitude daytime F2layer is well known. Extreme ultraviolet $(10.1 \mu \mathrm{m})$ solar radiation ionises atomic oxygen within the layer. The critical frequency foF2 is proportional to the maximum square root of the density of the free electrons in the F2-layer. The F2-layer is the upper, almost fully ionized part of the F-layer situated at altitudes from about $200 \mathrm{~km}$ to more than $500 \mathrm{~km}$ above the surface of the Earth. Mikhailov et al. (2007) concluded
Table 1. Correlation coefficient between foEnorm and foF 2 norm for quiet days with very large foEnorm-values

\begin{tabular}{lll}
\hline value of foEnorm & $\begin{array}{l}\text { correlation coefficient } \\
\text { between foE } \\
\text { and foF2 } 2 \text { norm }\end{array}$ & $\begin{array}{l}\text { error of } \\
\text { correlation } \\
\text { coefficient }\end{array}$ \\
\hline all data & 0.07 & 0.02 \\
abs (foE & 0.03 \\
abs (foEm $)>1$ & 0.13 & 0.05 \\
abs $($ foEm $)>1.5$ & 0.16 & 0.08 \\
\hline
\end{tabular}

that positive and negative variations of the foF2-frequency at geomagnetic quiet times in the F2-region are mainly due to the atomic oxygen density variations which are presumably the result of the vertical gas motion in the thermosphere, also including E-region heights. Mikhailov et al. (2007) did not find point-to-point correlations between the electron densities in the E- and F2-layer, but only a statistical correlation.

Investigating the critical frequency foF2, data obtained for the same earthquakes with magnitudes $M>5$ and depths of the epicentres $h<60 \mathrm{~km}$ which occurred at a distance $R<$ $2000 \mathrm{~km}$ from the vertical sounding station "Tashkent" are considered. For the study, a method analogous to the method of analysis of the foE-frequency (described in Sect. 2) is applied. The seasonal dependence is excluded and the function foF2 norm is constructed, which is normalized by the meansquare deviation. Again, the days with high solar activity and magnetic disturbances are excluded from the analysis. For foF2norm, the superposition of epoches method is also performed. First results of the analysis, obtained for all data (all seasons) are shown in Fig. 7. There, data for foF2 for the same daytime hours of the day $(-1)$ were available for 215 earthquakes. In the case of day $(-1)$, the average value of foF 2 norm increases and almost reaches the $95 \%$ level of reliability. Thus, one might suggest that the increase of the foF2-frequency is connected with the increase of the foEfrequency. Consequently, one might conclude that disturbances of the ionisation density are brought forward by the magnetic field. On the other side, the authors are inclined to assume that the variations obtained on the day $(-1)$ are random. In the case of winter-earthquakes, the increase of the foF2norm-value is rather small (see Fig. 7).

The correlation coefficient of the correlations between foEnorm and foF2norm, calculated taking the complete data into account, is generally low. Thereby, days with high geomagnetic activity mainly contribute to the growth of the coefficient. When investigating seismo-ionospheric effects, days with increased geomagnetic and solar activity are excluded from the analysis and a correlation coefficient of the quiet days $\left(\Sigma K_{p}<30, W<100\right)$ of 7 per cent is found. If one chooses from all the quiet days only those with very strong (positive or negative) foEnorm-values, one obtains a weak increase of the correlation coefficient with growing 


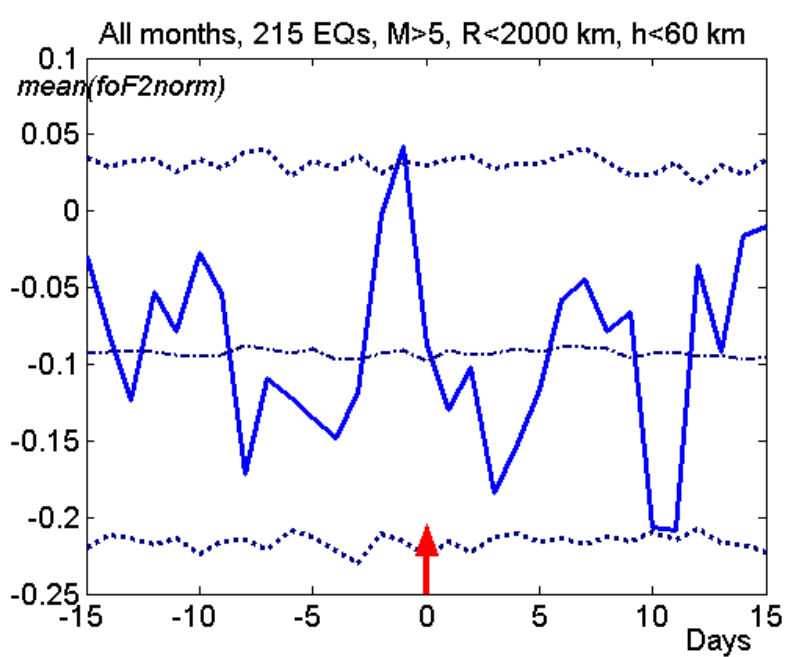

Fig. 7. Result of the superposition of epoches for foF 2 norm in case of the winter-earthquakes with $M>5, R<2000 \mathrm{~km}$, and $h<60 \mathrm{~km}$. The superposition time equals 30 days. The red arrow shows the day zero of the earthquakes. The mean value of foEnorm $(i)$ for the day $i$ during seismo-active times is presented by the solid blue line. The dash-dotted line designates the mean value of foF 2 norm for all data considered. The dotted lines show the $95 \%$-levels ( 2 std.) of reliability obtained using the method of modeling random processes.

foEnorm (see Table 1). Thus, one may conclude that only very large changes of the ionisation density in the E-layer influence the ionisation density in the F-region. However, such large ionisation densities in the E-layer, and correlated extreme values of foEnorm at the day $(-1)$, are a very rare phenomenon. Consequently, no synchronous growth of foEnorm and foF2norm could be observed.

\section{Discussion of the results and conclusions}

In the present work it is shown that in accordance to the observations of the vertical sounding station "Tashkent", the foE-frequency increases about one day before winterearthquakes with magnitudes $M>5$ and depths of the epicentre of $h<60 \mathrm{~km}$, which appear at distances of $R<$ $2000 \mathrm{~km}$ from the station. The reliability of the result is larger than $99 \%$. This finding is in agreement with observations by Ivanov-Kholodnyi and Tchertoprud (1998).

One may propose two mechanisms which might explain the foE-increase before earthquakes. First, the maximum increase of foE is obtained at distances of about $R \approx 1000 \mathrm{~km}$ from the epicentre. One may assume that this is caused by acoustic-gravity waves with periods of $1-3 \mathrm{~h}$. These waves propagate into the ionospheric E-layer at large distances of $1000 \mathrm{~km}$ to $1600 \mathrm{~km}$ from the epicentre (Brunelli and Namgaladze, 1988) and heat the E-layer by dissipation. When the temperature of the E-layer grows, the recombination coefficients decrease (Nikole, 1964) and, consequently, the ionisation density increases. The acoustic-gravity waves cross the stratosphere only during westerlies, which occur in winter (Danilov et al., 1987).

It might also be possible that foE grows as the E-layer is heated - and the recombination of ions decreases - during the seldom local upward propagation of neutral components of the atmosphere because of single acoustic pulses from the Earth's surface (Liperovsky et al., 2008) or, on the other hand, is due to disturbances of the electric field and mosaic-likely distributed atmospheric heating (Pulinets and Boyarchuk, 2004).

The mosaic-likely distributed atmospheric heating may be caused by radon emanation into the atmosphere before earthquakes and the formation of Frenkel areas of strong electric fields lasting some minutes, or some dozens of minutes. In this case, the disturbances are believed to propagate along the seismic fracture regions, and the mosaic-like processes are generated in the Earth's crust directly in the environment of the vertical sounding station.

Concerning the recombination of ions in the E-layer, it has yet to be mentioned that in this layer in daytime the main ions are $\mathrm{NO}^{+}$and $\mathrm{O}_{2}^{+}$ones. Besides, at E-layer altitudes the $\mathrm{N}_{2}^{+}$ions exist. But these ions have a short lifetime, and the $\mathrm{O}^{+}$. and $\mathrm{N}^{+}$-ions possess a low recombination coefficient. According to (Danilov, 1989), the effective recombination coefficient is approximately the sum of the recombination coefficients of $\mathrm{NO}^{+}$and $\mathrm{O}_{2}^{+}$, which are both proportional to the inverse electron temperature. So, in daytime during the increase of the temperature, the effective recombination coefficient decreases, and the degree of ionisation grows. Thus, also at growing temperature due to the dissipation of acoustic waves, the electron density and therefore also the critical frequency foE grow.

Concerning the foE-variations and possible correlated changes of the critical foF2-frequency before earthquakes, a first analysis showed only small correlations. Electron density changes in the F-layer might be preferably caused by acoustic and electric disturbances propagating, contrary to acoustic-gravity waves, mainly vertically upwards. But this is a topic of future research work.

Acknowledgements. The authors kindly thank the referees M. E. Contadakis and M. Rodkin for constructive comments.

Edited by: T. Maggipinto

Reviewed by: M. E. Contadakis and M. Rodkin 


\section{References}

Ambroz, P.: Statistical method of superposition of epochs, Bull. Astron. Inst. Czechosl., 30, 114-121, 1979.

Antonova, L. A., and Ivanov-Kholodnyi, G. S., and Tchertoprud, V. E.: Aeronomy of the E-layer, Janus, Moscow, 168 pp., 1996.

Brunelli, B. E. and Namgaladze, A. A.: Physics of the ionosphere, Moscow, Nauka, 527 pp., 1988.

Chapman, S.: The absorption and dissociative or ionizing effect of monochromatic radiation in an atmosphere on a rotating earth. Proc. Phys. Soc. Lond., 43, 26-45, 1931, 43, 483-501, 1931.

Danilov, A. D.: Popular aeronomy, Gidrometeoizdat, Leningrad, 271 pp., 1989.

Danilov, A. D., Kazimirovskiy, E. S., Vergasova, G. V., and Khatchikjan, G. Ja.: Meteorological effects in the ionosphere, Gidrometeoizdat, Leningrad, 1987.

Ivanov-Kholodnyi, G. S., Letshenko, L. N., and Odintzova, I. N.: Relationship between X-ray and ultraviolet radiations of solar flares in the ionization of the ionosphere E-region, Geomag. Aeronom. , 16, 246-250, 1976 (in Russian).

Ivanov-Kholodnyi, G. S. and Tchertoprud, V. E.: Distant reaction of the E-layer of the ionosphere on strong earthquakes, in: Short-time prediction of disastrous earthquakes using radiophysical earthbased-space methods, Institute for Physics of the Earth RAS, Moscow, 152-155, 1998.
Liperovskaya, E. V., Parrot, M., Bogdanov, V. V., Meister, C.-V., Rodkin, M. V., and Liperovsky, V. A.: On variations of foF2 and F-spread before strong earthquakes in Japan, Nat. Hazards Earth Syst. Sci., 6, 735-739, doi:10.5194/nhess-6-735-2006, 2006.

Liperovskaya, E. V., Biagi, P.-F., Meister, C.-V., and Rodkin, M. V.: foF2 seismo-ionospheric effect analysis: actual data and numerical simulations, Nat. Hazards Earth Syst. Sci., 8, 1387-1393, doi:10.5194/nhess-8-1387-2008, 2008.

Liperovskaya, E. V., Bogdanov, V. V., Biagi, P.-F., Meister, C.-V., Liperovsky, V. A., and Rodkin, M. V.: Day-time variations of foF2 connected to strong earthquakes, Nat. Hazards Earth Syst. Sci., 9, 53-59, doi:10.5194/nhess-9-53-2009, 2009.

Liperovsky, V. A., Pokhotelov, O. A., Meister, C.-V., and Liperovskaya, E. V.: Physical models of coupling in the lithosphereatmosphere-ionosphere system before earthquakes, Geomag. Aeronomy, 48 (6), 795-806, 2008.

Mikhailov, A. V., Depuev, V. H., and Depueva, A. H.: Synchronous $\mathrm{NmF} 2$ and NmE daytime variations as a key to the mechanism of quiet-time F2-layer disturbances, Ann. Geophys., 25, 483-493, 2007 , http://www.ann-geophys.net/25/483/2007/.

Nikole, M.: Aeronomy, Mir, Moscow, 278 pp., 1964.

Pulinets, S. A. and Boyarchuk, K. A.: Ionospheric precursors of earthquakes, Springer, Berlin, Heidelberg, New York, 315 pp., 2004. 